\title{
1. Einführung: Thema, Aufbau und Methodik der Untersuchung
}

Das deutsche Prosagedicht ist von seiten der Literaturwissenschaft seit jeher als äuBerst heikles literarisches Phänomen angesehen worden. Dies liegt zum einen am besonderen Charakter des Gattungsmodells, das tradierte Kommunikationsnormen infrage stellt und sich allen Versuchen, es als herkömmliche Textsorte behandeln zu wollen, hartnäckig widersetzt. Zum anderen gibt das eigenartige - durchaus auch qualitative - Gefälle, das zwischen den strahlkräftigen Mustern des poème en prose in Frankreich und den deutschsprachigen Ausprägungen des Genres besteht, Anlaß für Irritationen. Während die einschlägigen Texte Charles Baudelaires, Arthur Rimbauds und Stéphane Mallarmés fraglos zu den zentralen Gründungsurkunden der literarischen Moderne gerechnet werden können, ${ }^{1}$ geht den deutschen Gattungsbeispielen eine solche Aura ab, ja im internationalen Vergleich wirken die Texte verfahrenstechnisch oftmals konventionell. Welche kulturellen Faktoren für diese ästhetische Defizienz verantwortlich sind, blieb freilich bislang unerörtert. Die Forschung umging das Problem, indem sie den Gegenstand entweder marginalisierte oder ihn in seiner historischen Bedeutung verharmloste. ${ }^{2}$

Ziel der vorliegenden Untersuchung ist es daher, das deutsche Prosagedicht in den Kontext der gesamteuropäischen Gattungsentwicklung zu stellen. Dazu erweist es sich als unumgänglich nötig, zunächst die prägenden ausländischen Genrevorbilder zu untersuchen - und zwar vor allem im Hinblick darauf, welche Ansatzpunkte sie den Autoren im deutschsprachigen Raum boten, selbst daran anzuknüpfen. Bei der internationalen Ausbreitung des Prosagedichts handelt es sich nämlich um einen transkulturellen Rezeptionsvorgang, bei dem Vertextungsweisen von einem in ein anderes Literatursystem übertragen, dabei aber auch mehr oder weniger tiefgreifend transformiert werden. Eine besondere Rolle spielen in diesem Zusammenhang natürlich Übersetzungen; als mausgezeichnete Form interkultureller Kommunikation, die einen nachhaltigen Kulturkontakt und Kulturtransfer zwischen den Kulturen

1 Dies ist seit Hugo Friedrichs Studie über Die Strutur der modemen Lynik. Von Baudelaine bis zur Gegenwart (1956; als erweiterte Neuausgabe mit dem Untertitel Von der Mitte des newnzehnten bis zur Mitte des zanzigsten Jabrbunderts) opinio communis der Forschung.

2 Vgl. hierzu Kapitel I/2. 
herstellt" ${ }^{3}$, steuern sie nicht nur die Rezeption in hohem Maße, sondern geben auch wichtige Auskünfte über die Formen kultureller Selbst- und Fremdwahrnehmung. Auf dem Weg der Translation jedenfalls gelangte das - zuvor noch unbekannte Gattungsmodell nach Deutschland und Österreich und brachte dort einen Evolutionsprozeß in Gang, in dessen Verlauf sich eigenständige Textstrukturen herauszubilden begannen, die sich von denen des Ursprungslandes merklich unterscheiden. Zugleich setzten erste Rückkopplungsphänomene ein, welche nun ihrerseits die Genreentwicklung der Nachbarnationen beeinflußten.

Um nun die Transformationsvorgänge nachvollziehen zu können, die sich bei der Übertragung der französischen Gattungsinnovation in das deutsche Literatursystem ereigneten, gilt es, die Rahmenbedingungen des veränderten kulturellen Kontextes zu rekonstruieren, die dem Textmodell als spezifischer Art symbolischen Handelns seinen Stellenwert und seine Funktion zuweisen. Dabei zeigt sich einmal mehr, daß Gattungen als durch und durch kulturell geprägte Muster literarischer Kommunikation begriffen werden müssen. Wenn Kultur ein "Bedeutungsgewebe ${ }^{4}$ ("web of significanceu) darstellt und Literatur einen Teilbereich kultureller Praxis ${ }^{5}$ bildet, dann funktionieren Textsorten wie Steuerungselemente für die literarische Semantik, organisieren sie doch mehr oder weniger direkt durch die Schrift generierte bzw. generierbare Bedeutungen. Gattungen können demnach als Elemente einer kulturspezifischen Grammatik angesehen werden. Da sich die Zeichenhaftigkeit kulturellen Handelns auch in der Wahl der Muster literarischen Ausdrucks manifestiert, erweist sich die Entscheidung für oder gegen ein bestimmtes Genre als signifikant für die kulturelle Praxis eines bestimmten historischen Zeitraums. Gattungen erscheinen mithin als ästhetische Strukturierungsmatrizen, welche den symbolischen Status eines Textes entscheidend prägen, indem sie seine Rezeption regeln, seine soziale Verwendung steuern und seinen kulturellen Stellenwert bestimmen. ${ }^{6}$

Wie sich zeigt, fungiert das Prosagedicht im späten 19. Jahrhundert in ganz Europa als eine Projektionsfläche für das - mehr oder minder dringende ${ }^{7}-$ Bestreben

3 Andreas Poltermann: Literaturkanon - Medienereignis - Kultureller Text. Formen interkultureller Kommunikation und Übersetzung. In: Literaturkanon - Medienereignis - Kultureller Text. Formen interkultureller Kommunikation und Übersetzung. Hrsg. von A. P. Berlin: Erich Schmidt 1995 (= Göttinger Beiträge zur internationalen Übersetzungsforschung 10), S. 1.

4 Clifford Geertz: Dichte Beschreibung. Beiträge zum Verstehen kultureller Systeme [1983]. Frankfurt a.M.: Suhrkamp ${ }^{6} 1991$ (= suhrkamp taschenbuch wissenschaft 696), S. 9. Geertz rekurriert mit diesem Terminus auf die soziologische Theoriebildung Max Webers.

5 Vgl. Dietrich Harth: Die literarische als kulturelle Tätigkeit: Vorschläge zur Orientienung. In: Hartmut Böhme/Klaus R. Scherpe (Hrsg): Literatur und Kulturwissenschaften. Positionen, Theorien, Modelle. Reinbek: Rowohlt 1996 (= rowohlts enzyklopädie 575), S. 320-340.

6 Von hier aus könnten Vorstöße untemommen werden, die herkömmliche Textsortenforschung zu einem Teilbereich der Kultursemiotik weiterzuentwickeln.

7 Gründe hierfür werden in Kapitel $1 / 3$ erörtert. 
nach Gattungstransgression, wobei allerdings Modus und Funktion der damit intendierten Transgressionsakte erheblich voneinander abweichen. Das Genre wäre demnach zu deuten als jene "Institution ${ }^{8}$ im Literatursystem des späten 19. und frühen 20. Jahrhunderts, welcher die Aufgabe zukommt, generische Aspekte des ästhetischen Selbstreflexionsprozesses der Moderne im Medium der Form a auszutragen. Nicht zufällig operiert es an den Nahtstellen zwischen Lyrik und Prosa, sgebundener und rungebundener Sprache, ,Poesier und Nicht-Poesier, ${ }^{9}$ weil hier nicht nur die Frage zur Debatte steht, unter welchen Kriterien eine kategorisierende Zuordnung von Texten erfolgt, sondern auch die Berechtigung der hierarchisch abgestuften Zweiteilung des Literatursystems insgesamt werhandelt" ${ }^{10}$ werden kann. Insofern fungiert das Prosagedicht gewissermaßen als Schatten der (Vers-)Lyrik, der sichtbar zu werden beginnt, sobald die lyrischen Vertextungskonventionen als problematisch erkannt werden (und der allem Anschein nach solange weiterbestehen wird, wie die dichotomische Konstruktion des Literatursystems weiterexistiert).

Eine Studie zum Prosagedicht muß sich deshalb zentral mit dem Problem der Codierung von Poetizität auseinandersetzen, das in der Moderne in besonderem $\mathrm{Maß}$ virulent wird, da die Verabschiedung überzeitlicher ästhetischer Normen ein Vakuum erzeugt, das durch neu zu generierende - historisch wie kulturell variable Parameter literarischer Kommunikation erst wieder gefült werden muß. Da freilich nach dem Zerbröckeln einer transzendent oder auch anthropologisch verankerten Textontologie ein verbindlicher Konsens darüber, was denn nun als spoetischr gelten könne, nicht mehr herzustellen ist, kommt es zu einer Pluralisierung und Individualisierung künstlerischer Normen, welche die Lage recht unübersichtlich werden läßt. Zugleich aber bleibt jeder Ansatz einer subjektiven Neubegründung ästhetischer

8 Vgl. hierzu Wilhelm Voßkamp: Gattungen als literarisch-soziale Institutionen. (Zu Problemen sozial- und funktionsgeschichtlich orientierter Gattungstheorie und -historie.) In: Textsortenlehre - Gattungsgeschichte. Mit Beiträgen von Alexander von Bormann, Ulrich Fülleborn, Klaus W. Hempfer, Jost Hermand, Walter Hinck, Helmut Koopmann und W. V. Hrsg. von W. H. Heidelberg: Quelle \& Meyer 1977 (= medium literanur 4), S. 24-35; Harald Fricke: Norm und Abweichung. Eine Philosophie der Literatur. München: $C . H$. Beck 1981 (= Beck'sche Elementarbücher), S. 138-150; und Gottlieb Gaiser: Institutionen und Institutionalisierungsprozesse im System der Literatur. In: Sprachkunst 13 (1982), S. 269-281.

9 Es ist damit freilich noch lange nicht eine hybride Mischform bereits bestehender Gestaltungsweisen, als die man das neuartige Textmodell in der deutschen Literaturwissenschaft lange Zeit geme sehen wollte. So heißt es etwa bei Fülleborn, dem Hauptvertreter der germanistischen Prosagedichtforschung: "Was man hier erstrebte und terminologisch fixierte, war Lyrik, neuartige Lyrik, in ungebundener Rede«; Ulrich Fülleborn: Das deutsche Prosagedicht. Zu Theorie und Geschichte einer Gattung. München: Fink 1970, S. 10. Zum Begriff der "Verhandlung (mnegotiation«) in der neueren kulturwissenschaftlichen Theoriebildung vgl. vor allem Stephen Greenblatt: Verhandlungen mit Shakespeare. Innenansichten der englischen Renaissance [1988]. Aus dem Amerikanischen von Robin Crakkett. Frankfurt a.M.: Fischer Taschenbuch Verlag 1993 (= Fischer Taschenbuch 11001). 
Konzepte in doppelter Weise traditionsgebunden: Denn zum einen muß er sich mindestens negativ auf das überkommene System der Poetik beziehen, um sich davon abstoßen zu können, und zum anderen erfordert die Notwendigkeit intersubjektiver Verständigung zumindest ein gewisses $\mathrm{Maß}$ an Rückbezug auf die angestammten textuellen Ausdrucksmuster. Die Leitthese der vorliegenden Arbeit besteht nun darin, daß das Prosagedicht in diesem Zusammenhang eine historische Vorreiterrolle spielt und deshalb als paradigmatische Gattung der modernen Literatur bezeichnet werden kann. Tynjanovs Vorschlag folgend, der dazu aufgerufen hat, Literaturgeschichte als wdynamische Archäologie " ${ }^{11}$ zu betreiben, soll das Genre daher als textuelles Leitfossil der Moderne angesehen werden, das exemplarisch Auskunft geben kann über die ästhetischen Aporien des späten 19. und frühen 20. Jahrhunderts. Eine Geschichte dieser Textform ermöglicht also nicht nur eine querschnittartige Bestandsaufnahme des deutschen Literatursystems um 1900, sondern gibt auch einen Einblick in die textuellen Zirkulationsbedingungen der Moderne.

Wenn das Prosagedicht als Leitgattung der Moderne bezeichnet wird, dann heißt das natürlich nicht, daß es rein mengenmäßig den Zeitraum um 1900 dominieren würde. Auch soll damit nicht behauptet werden, daß die herausragenden literarischen Leistungen der Jahrhundertwende im Rahmen dieser Ausdrucksform sich ereignet hätten. Richtet man das Augenmerk auf die bloße Quantität der Texte oder betrachtet in erster Linie deren ästhetische Qualität, dann fällt das Ergebnis sogar reichlich ernüchternd aus. ${ }^{12}$ Obwohl das Genre durchaus breite Verwendung gefunden hat - die Gesamtheit seiner Erscheinungsformen läßt sich heute noch gar nicht zur Gänze überblicken -, und das bei Autoren nahezu aller Schichten literarisch

11 Jurij N. Tynjanov: Das Problem der Verssprache. Zur Semantik des poetischen Textes [1924]. Aus dem Russischen übersetzt, eingeleiter und mit Registern versehen von Inge Paulmann. München: Fink 1977 (= Theorie und Geschichte der Literatur und der schönen Künste. Texte und Abhandlungen 25), S. 45. Nach ihm haben dann vor allem Foucault sowie Aleida und Jan Assmann den Terminus aufgegriffen und ihn zum methodischen Leitbegriff ihrer Arbeit gemacht; vgl. Michel Foucaults Studien La naissane de la clinique. Une archéologie du regard médical (1963; dt.: Die Geburt der Klinik. Eine Archäologie des ärztlichen Blicks), Les mots et les choses. Une archéologie des scienies bumaines (1966; dt.: Die Ordnung der Dinge. Eine Archäologie der Humanwissenschaften) und Archéologie du savoir (1969; dt: Archäologie des Wissens) bzw. die einzelnen Bände der von Aleida und Jan Assmann herausgegebenen "Archäologie der literarischen Kommunikation«. Als wenig ergiebig erweist sich dagegen der Band von Jochen Hengst: Ansätze zu einer Archäologie der Literatur. Mit einem Versuch über Jahnn's Prosa. Stuttgart/Weimar: Metzler 2000 (= M \& P-Schriftenreihe für Wissenschaft und Forschung).

Damit wurde das - wohlgemerkt: deutschsprachige - Prosagedicht sowohl für empirisch orientierte Forschungsansätze im Rahmen etwa einer Sozialgeschichte der Literatur als auch für ambitionierte theoriegeleitete Textlektüren beispielsweise poststrukturalistischer Provenienz gleichermaßen uninteressant. Diese doppelte Inkompatilität mit jüngeren methodischen Leitkonzepten dürfte im übrigen einer der Gründe dafür sein, daß es bislang keine brauchbare Monographie über die Entwicklung der Gattung in Deutschland gab. 
ambitionierter Produktion, blieb es - gemessen an der Vielzahl der übrigen literarischen Gattungen - insgesamt gesehen doch eher ein Randphänomen. ${ }^{13}$ Ebensowenig dominiert es die poetologischen Debatten der Zeit. Wenn das Prosagedicht gelegentlich explizit Gegenstand der Erörterung wird, dann im Zusammenhang genereller Überlegungen zum Stellenwert der Lyrik in der Moderne oder zur Zukunft der Literatur insgesamt.

Der Erfolg eines historisch jungen Gattungskonzepts mißt sich freilich nicht nur an der Anzahl von publizierten Texten oder an seiner Präsenz in der öffentlichen Debatte, sondern vor allem auch daran, wie sehr die Autoren eines Literatursystems eine prinzipielle Auseinandersetzung mit diesem Vertextungsmuster als unumgängliche Verpflichtung ansehen - unabhängig davon, ob es dann tatsächlich genutzt wird oder nicht. Innerhalb einer "Kommunikationsgeschichte der Moderne ${ }^{14}$ ist mithin verstärkt danach zu fragen, welchen Stellenwert das neue Genre in der Konkurrenz bestehender Gattungen einnimmt und in welchem $\mathrm{Maß}$ es als immanenter Bezugspunkt auktorialer Poetik angesehen wird. Die eigentliche Bedeutung des Prosagedichts liegt denn auch in seiner Funktion als Katalysator der innerliterarischen Entwicklung. Es wirkt als Störelement, das zu einer Deformation der herkömmlichen literarischen Klassifikationsparameter führt und so eine Erneuerung des Formenspektrums und - in Konsequenz daraus - eine Umstrukturierung des Gattungssystems erzwingt. Indem das Prosagedicht die weitere Ausdifferenzierung literarischer Ausdrucksmuster in Gang bringt und vorantreibt, trägt es in erheblichem Maß zur Überwindung des vormodernen Textsortenkanons bei. ${ }^{15}$ Das Genre erweist sich

13 Siehe hierzu Lars Nylander: Prosadikt och modemitet. Prosadikt som gränsföreteelse i europeisk litteratur, med särskild inriktning pä Skandinavien 1880-1910. Stockholm/Stehag. Symposion Bokförlag 1990 (= Symposion Bibliothek)

14 Walter Schmitz: Erzählte Bilder: Zum Verschwinden des Auratischen in der Literatur der Moderne um 1900. In: Das Sprach-Bild als textuelle Interaktion, hrsg. von Gerd Labroisse und Dick van Stekelenburg. Amsterdam/Atlanta (Georgia): Rodopi 1999 (= Amsterdamer Beiträge zur neueren Germanistik 45), S. 216. Wichtige Vorarbeiten zu einer solchen hat besonders Sprengel vorgelegt; vgl. Peter Sprengel: Literatur im Kaiserreich. Studien zur Modeme. Berlin: Erich Schmidt 1993 (= Philologische Studien und Quellen 125); P. S./ Gregor Streim: Berliner und Wiener Moderne. Vermittlungen und Abgrenzungen in Literatur, Theater, Publizistik. Mit einem Beitrag von Barbara Noth. Wien/Köln/Weimar: Böhlau 1998 (= Literatur in der Geschichte - Geschichte in der Literatur 45); P. S.: Geschichte der deutschen Literatur von den Anfängen bis zur Gegenwart. Begründet von Helmut de Boor und Richard Newald. Bd. 9/I: Geschichte der deutschsprachigen Literatur 1870 1900: Von der Reichsgründung bis zur Jahrhundertwende. München: C.H. Beck 1998.

Bourdieu hat diesen Funktionsmechanismus kultureller Innovation als erster ausführlich beschrieben: "Auf dem Markt zu einem gegebenen Zeitpunkt einen neuen Produzenten, ein neues Produkt und ein neues Geschmackssystem durchzusetzen heißt, die Gesamtheit der unter dem Gesichtspunkt des Legitimitätsgrades hierarchisierten Produzenten, Produkte und Geschmackssysteme ein Stück weit in die Vergangenheit zu schieben.. Pierre Bourdieu: Die Regeln der Kunst. Genese und Struktur des literarischen Feldes [1992]. Übersetzt 
mithin weniger als diskursprägend denn als bedeutsamer Einflußfaktor für die literarische Praxis selbst. Als nahezu unumgänglicher Bezugspunkt mindestens für die Lyriker der Zeit wird es zu einer der treibenden Kräfte bei der Umgestaltung und Rekonstellierung des Gattungsensembles um 1900. Es funktioniert demnach wie eine Art Gravitationszentrum, das zwar durchaus auch ein breites Spektrum von Texten generiert, dessen epochale Wirkung aber in erster Linie an den Veränderungen der symbolischen Hierarchien innerhalb des Literatursystems und dessen diskursiver Redeordnung ablesbar ist.

Die Untersuchung wird sich denn auch weniger mit der Interpretation einzelner Texte befassen (wie dies Monographien jüngeren Datums bevorzugt getan haben ${ }^{16}$ ), sondern vielmehr die bei jedem Autor unterschiedliche Indienstnahme des Gattungsmodells Prosagedicht in den Mittelpunkt der Analyse rücken. Daß der Zuschnitt der einzelnen Kapitel vielfach personenzentriert gestaltet ist, resultiert aus der Einsicht, daß literarische Gestaltungsmuster sich nicht autonom entwickeln, sondern daß jedes Genre ein funktionales Element innerhalb eines auktorialen Poetikentwurfs darstellt und deshalb als wichtiger Signalfaktor innerhalb der Autor/Leser-Kommunikation ${ }^{17}$ verstanden werden muß. Zugleich lassen sich in der Nutzung einer Vertextungsform natürlich auch transpersonale Muster feststellen. Um solche übergreifenden Strukturen in den Blick zu rücken, widmen sich mehrere Kapitel Autorengruppen (Naturalisten, Expressionisten), an denen nicht zuletzt ihre regionale Ausprägung (Münchner Moderne, Wiener Moderne) interessiert. Dazwischen werden immer wieder themenzentrierte Abschnitte geschoben, deren Aufgabe es ist, ästhetische Wandlungsprozesse in threr jeweiligen kulturellen Ausprägung nachzuzeichnen. Denn nur vor dem Hintergrund der Umbrüche ästhetischer Kommunikation um 1900 kann die Entwicklung eines Texttyps, wie ihn das Prosagedicht darstellt, beschrieben werden.

Gattungsgeschichten traditionellen Zuschnitts isolieren ihren Gegenstand indes häufig aus dem systemischen Zusammenhang der Literaturentwicklung, wodurch die komplexen Wechselwirkungen mit benachbarten Textsorten aus dem Blick geraten,

von Bernd Schwibs und Achim Russer. Frankfurt a.M.: Suhrkamp 2001 (suhrkamp taschenbuch wissenschaft 1539), S. 257.

16 Vgl. vor allem Stefan Nienhaus: Das Prosagedicht im Wien der Jahrhundertwende. Altenberg - Hofmannsthal - Polgar. Berlin/New York: de Gruyter 1986 (= Quellen und Forschungen zur Sprach- und Kulturgeschichte der germanischen Völker N.F. 85), aber auch Comelia Ortlieb: Poetische Prosa. Beiträge zur modemen Poetik von Charles Baudelaire bis Georg Trakl. Stuttgart/Weimar: Metzler 2001 (= M \& P Schriftenreihe für Wissenschaft und Forschung).

17 Vgl. hierzu Wolfgang Bunzel: Rück-Wirkung: Goethes literarische Reaktionen auf die Rezeption seines Romans Die Leiden des jungen Wertherr. Eine historische Fallstudie als Baustein zu einer künftigen Theorie der Autor/Leser-Kommunikation. In: Bernhard Beutler/Anke Bosse (Hrsg.): Spuren, Signaturen, Spiegelungen. Zur Goethe-Rezeption in Europa. Köln/ Weimar/Wien: Böhlau 2000, S. 129-167. 
die im Prozeß der Konstitution und Transformation eines Genres eine entscheidende Rolle spielen. Will man zu einem bestimmten Zeitpunkt den symbolischen Stellenwert eines Textmodells ermitteln, dann wird man dessen Verflechtungen im Literatursystem der jeweiligen Kultur nachgehen müssen. Dies bedeutet aber nicht nur, die Austauschbeziehungen und Werthierarchien zu thematisieren, die im Ensemble der Genres von Belang sind; Aufmerksamkeit verdienen neben den Formen, in denen ein Gattungskonzept genutzt wird, auch - so paradox es klingen mag - die Umstände seiner Nichtnutzung, besonders dann, wenn diese Nichtnutzung ostentative Züge trägt. In die Analyse einbezogen werden deshalb auch Autoren, die sich nachweislich intensiv mit der Form des Prosagedichts auseinandergesetzt haben, selbst wenn dies zu keiner nennenswerten oder auch zu gar keiner Produktion von genrespezifischen Texten geführt hat. So hat beispielsweise Arno Holz das Genre regelrecht boykottiert; mit Hugo von Hofmannsthal und Rainer Maria Rilke wiederum gehören zwei der unbestritten wichtigsten Schriftsteller der Jahrhundertwende zur nicht kleinen Gruppe der lediglich sporadischen Nutzer. Ein derartiger Darstellungsfokus legt eine besondere Art der Materialanordnung nahe, und so stehen im folgenden neben Kapiteln, die sich mit Protagonisten der Genreentwicklung beschäfrigen (und die daher so auch in jeder Gattungsgeschichte herkömmlichen Typs stehen könnten), auch solche, die charakteristische Verschiebungen im Kunstsystem um 1900 erörtern bzw. den Blick auf Schlüsselfiguren der zeitgenössischen Literaturentwicklung richten. Dabei ließen sich im Fortgang der Argumentation gewisse Wiederholungen schlechterdings nicht vermeiden, doch wurde versucht, ihnen dort, wo sie unumgänglich sind, den Charakter einer Reprise zu geben, die ein bereits angeschlagenes Thema erneut aufgreift und mit veränderter Akzentsetzung erörtert.

Als weiteres Problem für die hier in Angriff genommene Untersuchung hat sich der Umstand erwiesen, daß die Fülle der um 1900 entstandenen Prosagedichte gegenwärtig bibliographisch noch gänzlich unerfaßt ist. Gesicherte Aussagen über die Nutzung des Genres durch heute weitgehend unbekannte Schriftsteller können daher momentan kaum getroffen werden. Doch auch wenn das Fehlen biographischer Informationen über die jeweiligen Verfasser, die Unkenntnis poetologischer Äußerungen und der mangelnde Einblick in Umfang und Struktur der CEuvres entsprechenden Analysen enge Grenzen setzen, wurde zumindest ansatzweise versucht, die Beschaffenheit dieser terra incognita zu sondieren. Wie eine systematische Recherche in den elektronisch aufbereiteten und allgemein zugänglichen Bestandskatalogen der deutschen Bibliotheken und eine stichprobenartige Überprüfung des gedruckten Gesamtverzeichnisses des deutschsprachigen Schrifttums ${ }^{18}$ ergibt, sind insgesamt nur sehr we-

18 Gesamtverzeichnis des deutschsprachigen Schriftums (GV) 1700-1910. Bearbeitet unter der Leitung von Peter Geils und Willy Gorzny. Bibliographische und redaktionelle Beratung: Hans Popst und Rainer Schöller. 160 Bde. München/New York/London/Paris: K.G. Saur 1979; (GV) 1911-1965. Hrsg. von Reinhard Oberschelp. Bearbeitet unter Lei- 
nige Publikationen erschienen, welche den Gattungsnamen oder ein direktes Derivat davon in der Titelei aufweisen. Unüberschaubar dagegen ist die Anzahl derjenigen Buchveröffentlichungen, die mit beliebten Synonymbegriffen wie sSkizze oder `Novelletter operieren, wobei freilich im Einzelfall immer erst nachgeprüft werden müßte, ob hier lediglich Kurzprosatexte im weitesten Sinn des Wortes oder tatsächlich Prosagedichte mit einem verifızierbaren Genresignalement vorliegen. Auch wäre zu eruieren, inwieweit die in Buchform versammelten Texte zuvor in Zeitschriften vorabgedruckt worden sind. Ein Abgleich der ermittelten Daten deutet jedenfalls darauf hin, daß viele Autoren, die mit Prosagedichten in Periodika vertreten sind, später keine entsprechenden Textsammlungen veröffentlicht haben. Man wird daher sagen müssen, daß die hier angestellte Studie in gewisser Weise nur die Spitze des Eisbergs erfaßt, den das Phänomen Prosagedicht tatsächlich darstellt. Allerdings bestätigen die Ergebnisse, die bei punktuellen Analysen bislang unerforschter Bereiche zutage gefördert werden konnten, die gewonnenen Befunde. Die hier getroffenen Aussagen werden deshalb durch eine erweitere Kenntnis des Quellenmaterials wohl allenfalls Ergänzungen, kaum aber grundlegende Korrekturen erfahren.

Was den Untersuchungszeitraum der Arbeit angeht, so wurde er bewußt auf die Jahrhundertwende beschränkt. Den Beginn der deutschsprachigen Gattungsentwicklung markiert dabei das Erscheinen des Bandes Adjutantenritte und andere Gedichte (1883), der ersten Buchveröffentlichung Detlev von Liliencrons. Nach wornı mußte notgedrungen eine willkürliche Grenze gezogen werden, um den Zeitraum der Untersuchung sinnvoll einzugrenzen. Eine Jahreszahl oder gar ein konkretes Datum kam dafür natürlich nicht infrage. Es lag vielmehr nahe, sich am Phasenverlauf des Genres zu orientieren. Wie die Verbreitung kultureller Novitäten unter den Bedingungen der Moderne generell vollzieht sich auch die des Prosagedichts im Rahmen eines charakteristischen Zirkulationsszyklus ${ }^{19}$, d.h. die Entwicklung des auf dem Weg kulturellen Transfers in das deutsche Literatursystem eingeschleusten Vertextungsmodells durchläuft charakteristische Etappen: Der schrittweisen Durchsetzung und zunehmenden Ausbreitung in einem bestehenden Gattungsensemble folgt die allmähliche sTrivialisierung, die durch ein Übermaß an Nutzung in Gang gesetzt wird; daran schließen sich Versuche an, den verlorenen Status der Textsorte zurückzuerobern. Spätestens mit dem Nachrücken aktuellerer Gestaltungsmuster geht das Genre dann endgültig in den Traditionsbestand existierender Ausdrucksformen ein gleichgültig, wie subversiv es einstmals auch gewesen sein mag. Wie sich im Fort-

tung von Willi Gorzny. Mit einem Geleitwort von Wilhelm Totok. 150 Bde. München: Verlag Dokumentation 1977.

19

Dieser auf charakteristische Entwicklungsphasen eines künstlerischen Gestaltungsmodus zielende Terminus lehnt sich an den Begriff des "Produktionszyllus» an, den Bourdieu ohne dies freilich explizit zu benennen - aus der ökonomischen Theoriebildung übemommen hat; vgl. Pierre Bourdieu: Die Regeln der Kunst. Genese und Struktur des literarischen Feldes, S. 229. 
gang der Analyse zeigt, ist dieser Punkt mit dem Expressionismus erreicht. Die Darstellung erstreckt sich daher bis in die zwanziger Jahre des 20. Jahrhunderts, als die erste und grundlegende Entwicklungsphase des Genres an ihr Ende gelangt. Was danach kommt, sind Reprisen, d.h. wiederholende und variierende Reinszenierungen bereits stattgehabter Funktionalisierungsmöglichkeiten, die zweifellos von beträchtlichem Interesse sein können, aber ein Ausgreifen der Untersuchung auf radikal veränderte Bedingungen des literarischen Systems erfordert und dabei nicht nur unweigerlich zu Redundanzen geführt, sondern auch die Kohärenz der Arbeit unnötig beeinträchtigt hätten.

Auch die Evolution von Gattungen folgt also - wie die anderer zu textuellen Mustern geronnener Symbolisierungsformen - einem spezifischen Entwicklungsverlauf, der von den allgemeinen Bedingungen kultureller Produktion in Gang gebracht und gesteuert wird. ${ }^{20}$ Wie sich herausstellt, differenzieren sie sich dabei wnach Maßgabe des genuin symbolischen Kredits, den sie innehaben und verleihen ${ }^{21}$, aus. Im Hinblick auf das Prosagedicht nun wird - bezogen auf den deutschsprachigen kulturellen Kontext - genau die zentrale Phase dieses generischen Ausdifferenzierungsprozesses nachgezeichnet. So läßt sich mitverfolgen, wie über rund vier Jahrzehnte hinweg ein ganzes Spektrum textueller Funktionalisierungsmöglichkeiten durchgespielt wird. Zudem liefert die Analyse der Art und Weise, wie sich das Genre auf dem literarischen Markt etabliert, wertvolle Aufschlüsse darüber, wie Konzepte von Autorschaft und literarischer Innovation, unter Bedingungen des forcierten Profilierungsdrucks der Moderne inszenatorische Umsetzung erfahren.

20 Die herkömmliche Strukturbildung in Phasen wie Aufstieg, Blüte gang freilich nimmt fälschlicherweise - wie die Analogisienung mit vegetativen Wachstumsprozessen zeigt - eine quasi natürlicher Gesetzmäßigkeit an, wo in Wirklichkeit soziale Determinationsmuster vorliegen, die historisch wandelbar sind.

21 Pierre Bourdieu: Die Regeln der Kunst. Genese und Struktur des literarischen Feldes, S. 190 . 
